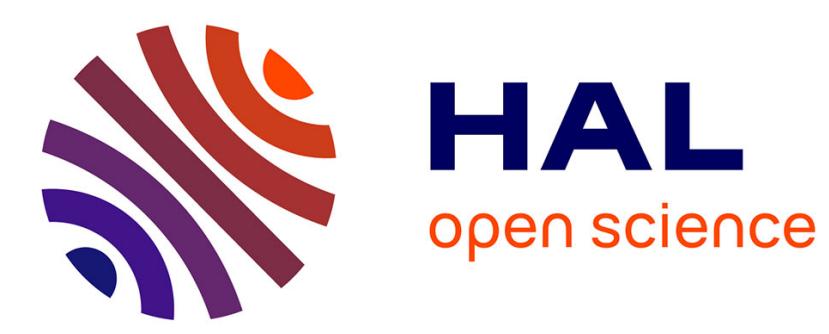

\title{
Van Hove singularities in Probability Density Functions of scalars
}

\author{
Patrice Meunier, Emmanuel Villermaux
}

\section{To cite this version:}

Patrice Meunier, Emmanuel Villermaux. Van Hove singularities in Probability Density Functions of scalars. Comptes Rendus Mécanique, 2007, 335 (3), pp.162. 10.1016/j.crme.2007.02.001 . hal00141770

\section{HAL Id: hal-00141770 \\ https://hal.science/hal-00141770}

Submitted on 27 Jun 2007

HAL is a multi-disciplinary open access archive for the deposit and dissemination of scientific research documents, whether they are published or not. The documents may come from teaching and research institutions in France or abroad, or from public or private research centers.
L'archive ouverte pluridisciplinaire HAL, est destinée au dépôt et à la diffusion de documents scientifiques de niveau recherche, publiés ou non, émanant des établissements d'enseignement et de recherche français ou étrangers, des laboratoires publics ou privés. 


\title{
Van Hove Singularities in Probability Density Functions of Scalars
}

\author{
P. Meunier, E. Vilermaux ${ }^{\dagger}$, \\ Université de Provence and CNRS, Institut de Recherche sur les Phénomènes Hors Équilibre, 49 rue \\ Frédéric Joliot-Curie, 13384 Marseille Cedex 13, France \\ ${ }^{\dagger}$ Also at: Institut Universitaire de France.
}

\begin{abstract}
A general theory for the Probability Density Function (PDF) of a scalar stirred in an axisymmetric time-dependent flow is derived. This theory reveals singularities, discontinuities and cusps occurring as soon as the spatial gradient of the scalar concentration vanishes somewhere in the field. These singularities are similar to the Van Hove singularities obtained in the density of vibration modes of a crystal. This feature, ubiquitous in convectiondiffusion problems, is documented experimentally for the mixing of a dye in a Lamb-Oseen vortex.

Key words: mixing, singularities, PDF

Résumé

Singularités de Van Hove dans les densités de probabilité d'un scalaire - Nous dérivons une théorie générale pour la Densité de Probabilité (PDF) d'un scalaire étiré dans un champ de vitesse axisymmétrique et dépendant du temps. Cette théorie révèle des singularités (discontinuités et cusps), qui apparaissent dès lors que le gradient spatial du scalaire s'annule en un endroit du champ. Ces singularités sont similaires aux singularités de Van Hove obtenue pour la densité des modes de vibration d'un cristal. Ce phénomène, omniprésent dans les problèmes de diffusion-convection, est documenté expérimentalement pour le mélange d'un colorant dans un vortex de Lamb-Oseen.
\end{abstract}

Mots-clés: mélange, singularités, PDF

The interplay between molecular diffusion and simple deformation fields is a classical problem. It is solved in closed form in a variety of situations such as the saddle point flow, the simple shear in two dimensions $[1,2,3]$, in three dimensions [4], and in the axisymmetric point vortex $[5,6]$ or spreading vortex flow $[7,8]$. In particular, simple situations are liable of a complete description of the overall Probability Density Function (PDF) of the scalar concentration from the Fourier equation [9].

Email address: meunier@irphe.univ-mrs.fr (P. Meunier). 


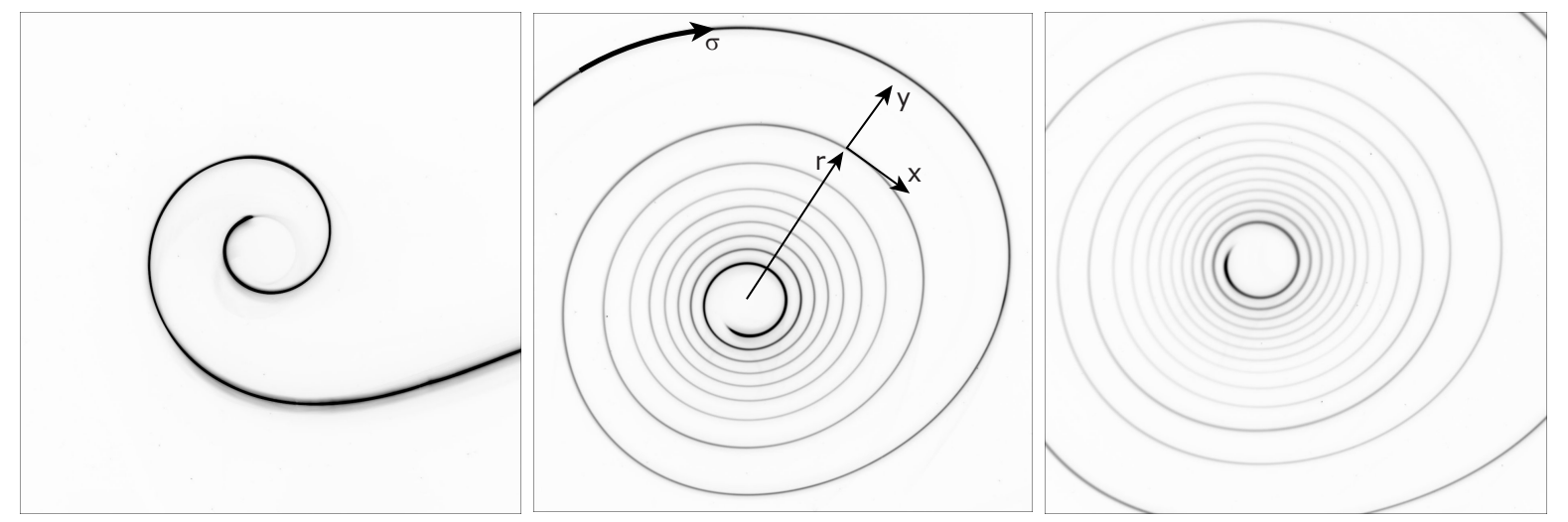

Fig. 1. Visualisation of the spiral formed by a dye lamellae wrapping around a Gaussian vortex. Images are obtained at $t=5 \mathrm{~s}, t=50 \mathrm{~s}$ and $t=95 \mathrm{~s}$ and cover $10 \times 10 \mathrm{~cm}^{2}$ for a vortex of circulation $\Gamma=17 \mathrm{~cm}^{2} \mathrm{~s}^{-1}$.

Here, we discuss the condition under which the PDF presents a singularity. The phenomenon we analyse first is illustrated on Fig. 1. A lamellae of weakly diffusive dye $\left(D=10^{-9} \mathrm{~m}^{2} / \mathrm{s}\right)$ with initial concentration $c_{0}$ and width $s_{0} \approx 2 \mathrm{~mm}$ is deposited in the velocity field of an axisymmetric, time-dependent vortex. The azimuthal velocity profile is Gaussian, that of a Lamb-Oseen vortex [10]

$$
v_{\theta}(r, t)=\frac{\Gamma}{2 \pi r}\left[1-\exp \left(\frac{-r^{2}}{a_{0}^{2}+4 \nu t}\right)\right]
$$

with a circulation $\Gamma=17 \mathrm{~cm}^{2} / \mathrm{s}$ and initial core size $a_{0}=1.3 \mathrm{~cm}$. The lamellae first rolls-up around the vortex (Fig. 1a), stretches and, conversely, gets thinner in its transverse direction. Diffusion is hasten by this stretching motion [9] and indeed, Fig. 1(b) shows that the dye concentration is smaller where the elongation is higher, i.e. at intermediate radii. Close to the vortex center in solid body rotation, and far from the vortex center where the velocity magnitude is small and so is the stretching rate, the dye still bears a concentration close to the initial one. The process goes on until at late stages (Fig. 1c), the dye has vanished in the diluting medium.

Compared to our first contribution on the topic [9], the set-up has been modified to allow the study of the late stages. Previously, the vortex would experience an axial destabilization 15 seconds after its formation due to the end effects. Here, the fluid is linearly stratified, stabilizing the vortex for 2 to 3 minutes. The dye is now injected by slowly translating a thin iron wire coated with fluoresceine, forming a vertical sheet of dye aligned with the vortex axis. A horizontal laser sheet allows the visualisation of a field crosssection, which is imaged from below (Fig. 1) using a Kodak 8 bits $2048^{2}$ pixels CCD array providing a 12 pixels $/ \mathrm{mm}$ resolution. While our theory was limited to a stationary point vortex $v_{\theta}=\Gamma / 2 \pi r$, we extend it here to the general case of a time-dependent axisymmetric flow as in Eq. 1. Computing the stretching of a length element $d r$ and using incompressibility, the distance between two meterial points in the direction perpendicular to the lamellae (striation thickness) is $s(r, t)=s_{0}\left[1+\left(r \int_{0}^{t} d t \partial\left(v_{\theta} / r\right) / \partial r\right)^{2}\right]^{-1 / 2}$, where $s_{0}$ is the initial lamellae thickness. In a frame of reference $(O, x, y)$ tangent to the lamellae with $y$ pointing outwards, the convection-diffusion equation for the dye concentration $c$ amounts to a pure diffusion equation $\partial c / \partial \tau=\partial^{2} c / \partial \xi^{2}$, when the transverse coordinate $y$ has been rescaled by the current striation thickness $(\xi=y / s(t))$ and time $t$ has been rescaled by the current diffusion time $\left(d \tau=D d t / s^{2}\right)$. The dimensionless time $\tau$ is

$$
\tau(r, t)=\frac{D t}{s_{0}^{2}}+\frac{D r^{2}}{s_{0}^{2}} \int_{0}^{t}\left(\int_{0}^{t^{\prime}} \frac{\partial\left(v_{\theta} / r\right)}{\partial r} d t^{\prime \prime}\right)^{2} d t^{\prime}
$$




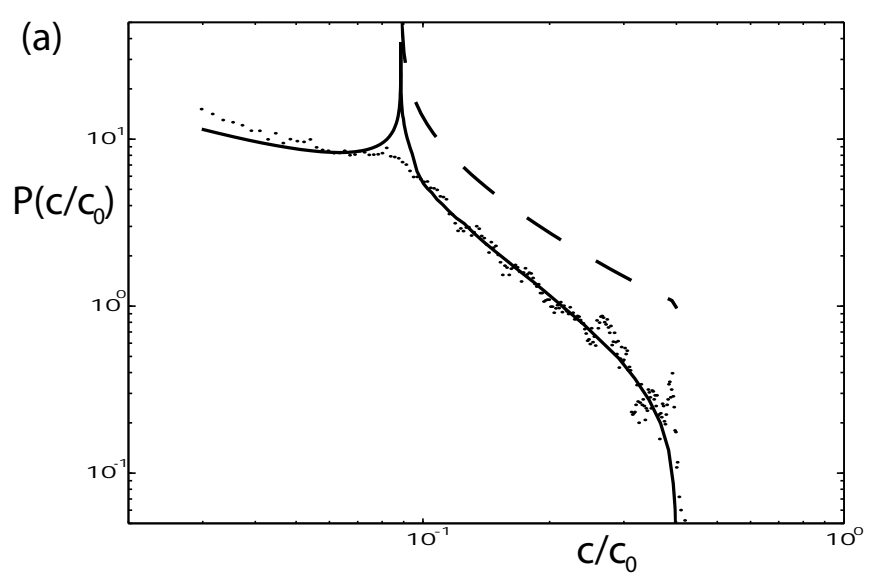

(b)

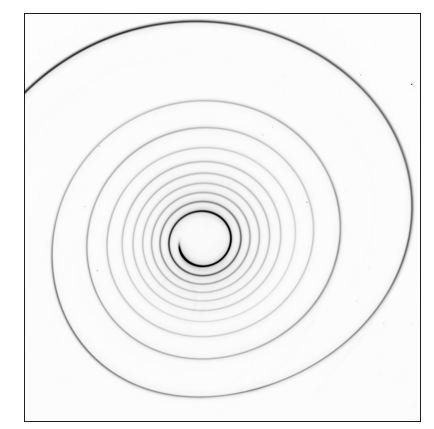

Fig. 2. (a) Probability density function of the concentration $c$ and (b) concentration field at $t=60 \mathrm{~s}$. . The solid line corresponds to the full model (4) and the dashed line to the approximation (6) using the maximal concentration.

At late stages, the dye concentration has a Gaussian profile in the transverse direction, with a maximum concentration $c_{M}(r, t)=c_{0} \operatorname{erf}(1 / 4 \sqrt{\tau})$ depending on $\tau$ only

$$
c(r, \xi, t) \approx c_{0} \operatorname{erf}\left(\frac{1}{4 \sqrt{\tau(r)}}\right) \exp \left(\frac{-6 \xi^{2}}{1+24 \tau(r)}\right)
$$

The PDF of such a spatial profile is easily obtained by integration over $r$ in the range where the maximum concentration $c_{M}(r)$ is larger than a given concentration level $c: P(c)=2 s_{0} \int_{c_{M}(r)>c}|\partial c / \partial \xi|^{-1} d r / A$ where $A$ is a normalising constant equal to the surface area on which the PDF is calculated. Expressing $\partial c / \partial \xi=-12 \xi c /(1+24 \tau)$, and inverting the function $c(\xi)$, we find the general solution

$$
P(c)=\frac{2 s_{0}}{c A} \int \max \left(0, \frac{\tau(r)+1 / 24}{\log [\operatorname{erf}(1 / 4 \sqrt{\tau(r)})]-\log \left[c / c_{0}\right]}\right)^{1 / 2} d r
$$

The function ' $\max (0,$.$) ' stands for the integrand to vanish when the maximum concentration c_{M}(r)$ is smaller than $c$; this allows the integration to be performed on the entire lamellae length at any time.

Defining the curvilinear abscissa $\sigma$ along the lamellae (Fig.1), this formula is extended to any twodimensional flow provided the stretching rate $\gamma(\sigma, t)=-[d s(\sigma) / d t] / s(\sigma)$ of all the lamellae elements is known at any time along their Lagrangian trajectory. The integral is computed over $\sigma$ instead of $r$ for axisymmetric flows, giving

$$
\tau(\sigma, t)=\frac{D}{s_{0}^{2}} \int_{0}^{t} \exp \left(2 \int_{0}^{t^{\prime}} \gamma\left(\sigma, t^{\prime}\right) d t^{\prime \prime}\right) d t^{\prime}
$$

Prediction (4) is plotted in Fig. 2 and shows good agreement with the measured distribution. The general solution (4) can be further simplified when $\tau(r)$ is rapidly varying with $r$. By a change of variable $u=c_{M}(r) / c$, the integral is modified into $\int_{1}^{\infty} c d u \sqrt{\tau+1 / 24} / \sqrt{\log (u)} \partial c_{M} / \partial r$. Since this function diverges for $u=1$, the other terms can be replaced by their values in $u=1$, which corresponds to $c_{M}(r)=c$, giving

$$
P(c) \approx \frac{\sqrt{\tau+1 / 24}}{\partial c_{M} / \partial r},
$$

a result easily obtained by retaining the maximum of the concentration at each radius only in computing the PDF, with a weigth equal to the transverse size of the Gaussian profile $\sqrt{\tau+1 / 24}$, giving $P\left(c_{M}\right) d c_{M}=$ 
(a)
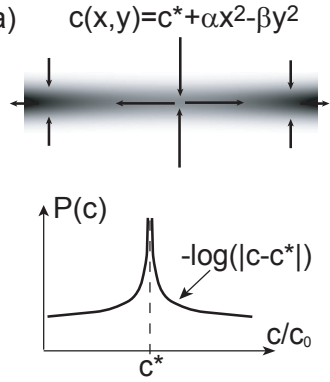

(b) $\quad c(x, y)=c^{*}-\alpha x^{2}-\beta y^{2}$

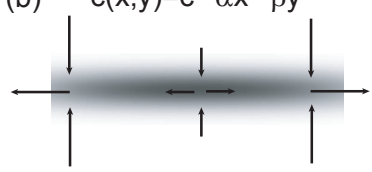

${ }_{\uparrow} P(\mathrm{c})$

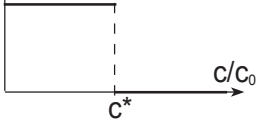

Fig. 3. Schematic of the singularities occurring in the scalar PDF of a 2D flow. (top) Pseudo-color plot of the scalar distribution $c(x, y)$. (bottom) The PDF is computed using $P(c)=4 \int_{0}^{R / \sqrt{\alpha}}|\partial c / \partial y|_{y_{0}(x)}^{-1} d x$, with $y_{0}(x)=\sqrt{c^{*}-c+\alpha x^{2}} / \sqrt{\beta}$ for (a) and $y_{0}(x)=\sqrt{c^{*}-c-\alpha x^{2}} / \sqrt{\beta}$ for (b).

$\sqrt{\tau+1 / 24} d r$. This ansatz, also plotted in Fig. 2, reproduces the shape of the PDF correctly, and is off by a factor 2 in amplitude. This discrepancy is a normalization artifact due to the fact that the PDF of the maximal concentration in Eq. 6 does not represent levels smaller than the minimum of $c_{M}(r)$. This PDF is thus restricted to $c>0.09 c_{0}$, where the minimum of the maximal concentration over the dye lamellae is at that instant of time. This approximation is thus all the more valid that the lamellae is deposited initially far from the vortex center, in a region where $\tau(r)$ is systematically decreasing with $r$. There, this ansatz moreover predicts that $P(c) \sim t^{-3 / 4} c^{-3 / 2}$, asymptotically [9].

These predictions seem to be good enough when $\tau(r)$ and thus $c_{M}(r)$ are monotonic functions of the radius. However, both predictions reveal a singularity when $\tau(r)$ has an extremum, since formulas (4) and (6) diverge when $\partial c_{M} / \partial r$ vanishes. We now focus on these critical points.

Figure 2 shows that the theoretical PDF presents a singularity at $c^{*} / c_{0}=0.09$. Before commenting on the experimental distribution, let us first recognize that such a phenomenon will obviously appear as soon as the gradient of the scalar $\partial c / \partial \mathbf{r}$ vanishes somewhere in the concentration field. This was already noted by Van Hove [11] for the density of the elastic vibration frequencies of a crystal (density of modes). Depending on the shape of the dispersion relation $\omega(k)$ in the crystal, the density of modes $D(\omega)$ has a singularity where the group velocity $\partial \omega / \partial k$ vanishes. It is in fact a very general feature, arising for any calculation of a density distribution. We recall here the different types of singularities, corresponding in our case to the different topologies of the concentration profile where these events occur. Let us examine first concentration fields in two dimensions.

(i) First, consider a lamellae whose concentration profile presents a $2 \mathrm{D}$ saddle point: locally, the scalar concentration is equivalent to $c(x, y)=c^{*}+\alpha x^{2}-\beta y^{2}$ with $\alpha$ and $\beta$ positive. This case is shown schematically on Fig. 3(a) and can be obtained if the stretching is maximum somewhere along the lamellae. For $c<c^{*}$ the PDF is computed by integrating on a square surface of size $(R / \sqrt{\alpha}, R / \sqrt{\beta}): P(c)=4 \int_{0}^{R / \sqrt{\alpha}}|\partial c / \partial y|^{-1} d x$, and by expressing $y$ as a function of $c$ and $x: P(c)=$ $2 \operatorname{Arcsinh}\left(R / \sqrt{c^{*}-c}\right) / \sqrt{\alpha \beta}$. The PDF is thus equivalent to $-\log \left(c^{*}-c\right)$ close to the critical level $c^{*}$. The same scaling can be obtained for $c>c^{*}$ and the PDF thus presents a logarithmic singularity at $c=c^{*}$ in that case.

(ii) Second, consider the opposite case where the concentration has a local maximum on the lamellae, as can be realized if there is a minimum of stretching along the lamellae, as shown schematically on Fig. 3(b). The scalar concentration is locally equivalent to $c(x, y)=c^{*}-\alpha x^{2}-\beta y^{2}$. The PDF is equal to zero for $c>c^{*}$ and equal to $\pi / \sqrt{\alpha \beta}$ for $c<c^{*}$. The PDF is thus discontinuous as soon as the scalar concentration profile has a maximum.

(iii) Third, the concentration profile presents a local minimum. This is not likely to happen in two 

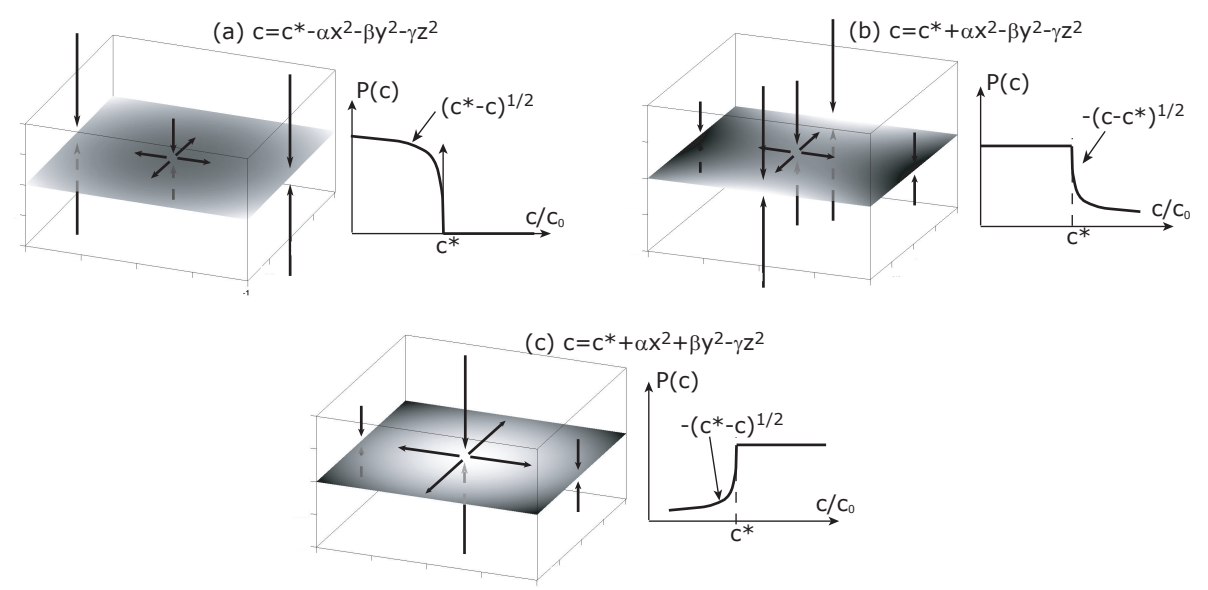

Fig. 4. Schematic of the singularities occurring in the scalar PDF of a 3D flow. (left) Pseudo-color plot of the scalar distribution at the center of the $3 \mathrm{D}$ sheet. (right) The PDF is calculated using $P(c)=8 \int_{0}^{R / \sqrt{\alpha}} \int_{0}^{R / \sqrt{\beta}}|\partial c / \partial z|_{z_{0}(x, y)}^{-1} d x d y$, with $z_{0}(x, y)$ defined such that $c\left(x, y, z_{0}\right)=c$.

dimensions since the topology is rather that of an elongated lamellae, with a transverse concave concentration profile. However, the PDF can be computed in this case also and is found to be discontinuous ( 0 for $c<c^{*}$ and $\pi / \sqrt{\alpha \beta}$ for $c>c^{*}$ ).

The PDF of a concentration field in two-dimensions can thus present logarithmic singularities or discontinuities at finite times i.e. as soon as the concentration profiles are affected by diffusion. One understands now why this divergence is rendered on the experimental distribution (Fig. 2) as a weak cusp only. This is primarily due to the finite size resolution of the digital images from which the concentration field is extracted. The logarithmic singularity comes from a very restricted area around a single point, the one with $c=c^{*}$. The second reason comes from the finite binning of the concentration levels, which enlarges and smoothes the singularity. However, this singularity, which comes from a maximum of the stretching along the spiral does exist (as clearly seen on Fig. 1(b)) and would be all the more resolved that the uncertainty in the definition of the concentrations, and the spatial resolution are sharp enough.

Consider now three-dimensional flows, where the scalar is usually found in the form of sheets as a result of various turbulent or chaotic motions $[12,13,14]$. These are shown schematically in Fig. 4 lying in the $(x, y)$ plane. A singularity in the corresponding concentration PDF may again appear when the concentration gradient $\partial c / \partial \mathbf{r}$ vanishes. There are thus four different cases depending on whether the concentration profile has a maximum or a minimum in each direction.

(i) The first case corresponds to a concentration profile with a maximum of concentration: it is locally equivalent to $c(x, y, z)=c^{*}-\alpha x^{2}-\beta y^{2}-\gamma z^{2}$ (with $\alpha, \beta$ and $\gamma$ positive). This can be obtained if the stretching has a local minimum (see Fig. 4a). The PDF, computed on a volume of radius $R$ is equal to $\pi \sqrt{c^{*}-c} / \sqrt{\alpha \beta \gamma}$ for $c<c^{*}$ and vanishes for $c>c^{*}$. The PDF presents a cusp with an infinite (negative) slope for $c<c^{*}$, as indicated on Fig. 4(a).

(ii) The second case corresponds to a concentration profile locally equivalent to $c(x, y, z)=c^{*}+\alpha x^{2}-$ $\beta y^{2}-\gamma z^{2}$, which is obtained if the stretching of the sheet is maximum along $y$ and minimum along $x$ (see Fig. 4b). The PDF is equal to $\pi\left(\sqrt{\gamma} R-\sqrt{c-c^{*}}\right) / \sqrt{\alpha \beta \gamma}$ for $c>c^{*}$ and to $\pi R / \sqrt{\alpha \beta}$ for $c<c^{*}$. The PDF presents a cusp with an infinite slope for $c>c^{*}$, as indicated on Fig. 4(b).

(iii) The third case corresponds to a concentration profile locally equivalent to $c(x, y, z)=c^{*}+\alpha x^{2}+$ $\beta y^{2}-\gamma z^{2}$, which is obtained if the stretching of the sheet has a maximum (see Fig. 4c). The PDF is equal to $\pi\left(\sqrt{\gamma} R-\sqrt{c^{*}-c}\right) / \sqrt{\alpha \beta \gamma}$ for $c<c^{*}$ and to $\pi R / \sqrt{\alpha \beta}$ for $c>c^{*}$. The PDF presents a 
cusp with an infinite slope for $c<c^{*}$, as indicated on Fig. 4(c).

(iv) Finally, the fourth case would correspond to a local minimum concentration as $c(x, y, z)=c^{*}+\alpha x^{2}+$ $\beta y^{2}+\gamma z^{2}$. It is not likely to be very frequent in 3D flows where the scalar has rather the topology of a sheet with a transverse concave concentration profile. However, the PDF can be calculated in that case too and is equal to $\pi \sqrt{c-c^{*}} / \sqrt{\alpha \beta \gamma}$ for $c>c^{*}$ and vanishes for $c<c^{*}$. The PDF again presents a cusp with an infinite slope for $c>c^{*}$.

As in two dimensions and for the same reason, the PDF of a concentration field in three-dimensions can thus present a cusp with an infinite slope each time the gradient of the scalar vanishes. This algebraic divergence is weaker than the logarithmic one in two dimensions.

The singularities are prominent in simple fields, as the one generated by a Gaussian vortex in the present experiment, where the concentration gradient vanishes at one location in the field only. In a complex flow stirred randomly, the number of these singularities should increase with time as the scalar field is distorted by the underlying motion. Indeed, the more complex the spatial scalar distribution, the more likely it will contain saddle points and maxima. However, these singularities will occur at an increasing number of different critical concentration levels $c^{*}$, at an increasing number of spatial locations in the flow [15]. The scalar PDF, initially presenting one, then two, then a large collection of singular cusps will progressively built-up in a continuous distribution, made of a continuous spectrum of singularities. Being rooted in the local topology of the field, these singularities are robust and may be found in 3D flows as well.

\section{References}

[1] A. Leveque, M., Les lois de la transmission de la chaleur par convection, Ann. Mines 13 (1928) 201-239.

[2] W. E. Ranz, Application of a stretch model to mixing, diffusion and reaction in laminar and turbulent flows, AIChE J. 25 (1979) 41-47.

[3] H. K. Moffatt, Transport effects associated with turbulence with particular attention to the influence of helicity, Rep. Prog. Phys. 46 (1983) 621-664.

[4] E. Villermaux, H. Rehab, Mixing in coaxial jets, J. Fluid Mech. 425 (2000) 161-185.

[5] P. B. Rhines, W. R. Young, How rapidly is a passive scalar mixed within closed streamlines, J. Fluid Mech. 133 (1983) 133-145.

[6] P. Flohr, J. C. Vassilicos, Accelerated scalar dissipation in a vortex, J. Fluid Mech. 348 (1997) 295-317.

[7] F. E. Marble, Mixing, diffusion and chemical reaction of liquids in a vortex field, in: M. Moreau, P. Turq (Eds.), Chemical Reactivity in Liquids: Fundamental Aspects, 1988, pp. 581-596, plenum.

[8] K. Bajer, A. P. Bassom, A. D. Gilbert, Accelerated diffusion in the centre of a vortex, J. Fluid Mech. 437 (2001) 395-411.

[9] P. Meunier, E. Villermaux, How vortices mix, J. Fluid Mech. 476 (2003a) 213-222.

[10] P. Meunier, T. Leweke, Analysis and optimization of the error caused by high velocity gradients in PIV, Exp. Fluids 35 (2003b) 408-421.

[11] L. Van Hove, The occurrence of singularities in the elastic frequency distribution of a crystal, Phys. Rev. 89 (6) (1953) 1189-1193.

[12] B. J. Cantwell, On the behavior of velocity gradient tensors invariats in direct numerical simulations of turbulence, Phys. Fluids A 5 (8) (1993) 2008-2013.

[13] K. A. Buch Jr, W. J. A. Dahm, Experimental study of the fine-scale structure of conserved scalar mixing in turbulent shear flows. part 1. sc $\gg 1$, J. Fluid Mech. 317 (1996) 21-71.

[14] G. O. Fountain, D. V. Khakhar, J. M. Ottino, Visualization of three-dimensional chaos, Science 281 (1998) $683-686$.

[15] C. H. Gibson, Fine structure of scalar fields mixed by turbulence. i. zero-gradient points and minimal gradient surfaces, The Physics of Fluids 11 (11) (1968) 2305-2315. 